\title{
Should selection criteria for HCC be the same (or different) between LDLT and DDLT?
}

\author{
Flavia Feier ${ }^{1,2}$ \\ 'Liver Transplantation Department, Hospital de Clinicas de Porto Alegre, Porto Alegre 90035-903, Brazil. \\ ${ }^{2}$ Pediatric Liver Transplantation Department, Irmandade Santa Casa de Porto Alegre, Porto Alegre 90020-090, Brazil. \\ Correspondence to: Dr. Flavia Feier, Liver Transplantation Department, Hospital de Clinicas de Porto Alegre, Rua Ramiro Barcelos \\ 2350, Porto Alegre 90035-903, Brazil. E-mail: flavia.feier@gmail.com
}

How to cite this article: Feier, F. Should selection criteria for HCC be the same (or different) between LDLT and DDLT? Hepatoma Res 2020;6:75. http://dx.doi.org/10.20517/2394-5079.2020.68

Received: 19 Jul 2020 First Decision: 8 Sep 2020 Revised: 9 Sep 2020 Accepted: 14 Sep 2020 Published: 6 Nov 2020

Academic Editor: Nobuhisa Akamatsu Copy Editor: Cai-Hong Wang Production Editor: Jing Yu

\begin{abstract}
Since the Milan Criteria (MC) were adopted in many countries as the allocation policy criteria for patients with hepatocellular carcinoma to be transplanted, many groups started to expand it to provide a chance for patients with tumors outside the MC who could achieve similar survival rates. With the scarcity of deceased donors, Asian countries improved the results with living donor liver transplantation, allowing patients outside $M C$ to be transplanted with a living donor. Newer prognostic models and a more profound understanding of tumor behavior are targeting better patient selection. Currently, patients are unevenly selected for liver transplantation and mostly separated into those fulfilling the MC and transplanted with a deceased donor and those with expanded criteria and transplanted with a living donor. In this paper, insight is brought into this debate.
\end{abstract}

Keywords: Living donor, hepatocellular carcinoma, alpha-fetoprotein

Liver transplantation (LT) is considered a curative treatment for patients with hepatocellular carcinoma (HCC) not amenable to surgical resection or ablative curative therapies. HCC was the indication for LT in $20.4 \%$ of the recipients in the USA in $2018^{[1]}, 14.4 \%$ in Europe ${ }^{[2]}$ and $7.6 \%$ and $19 \%$ for patients transplanted with deceased donors and living donors, respectively, in Japan ${ }^{[2]}$.

To provide a real chance for these patients to be transplanted with a deceased donor, exception points (outside the MELD score) have been given all over the world. However, in countries with organ shortage,

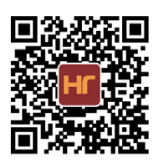


living donor liver transplantation (LDLT) has progressively expanded as the best alternative for such patients. In Asian countries, it is the most prevalent form of LT - 95\% of LT in Japan ${ }^{[2]}$ as opposed to $4.4 \%$ in the USA ${ }^{[1]}$, with excellent outcomes in both recipients and donors ${ }^{[3,4]}$. Certainly, donor safety is an ongoing concern, as a healthy individual will undergo a major surgical procedure. However, current morbidity and mortality rates following living liver donation have been reported to be $15 \%-25 \%$ and $0.5 \%$, respectively ${ }^{[5]}$. Driven by these results, the indications for LDLT in HCC patients were gradually expanded. The premise was that the survival benefit potentially achieved for those patients, within these expanded criteria, would not compromise the liver transplant allocation policy.

The inclusion criteria for HCC patients has dominated the scientific debate in the last years. Decades ago, the results from Mazzafero's group helped establish criteria to indicate LT for patients with HCC. The so-called Milan Criteria (MC) (single tumor $\leq 5 \mathrm{~cm}$, or less than 3 tumors $\leq 3 \mathrm{~cm}$ without major vessel involvement) reported a post-LT 4 -year survival of $75 \%$ for patients meeting these criteria ${ }^{[6]}$. This expected survival was comparable to that of patients transplanted for other indications, justifying organ allocation for patients with HCC.

However, several centers around the world considered the MC to be too strict, possibly excluding patients that still could benefit from LT. The University of California San Francisco expanded the criteria to a single nodule with a maximum diameter of $65 \mathrm{~mm}$, or two or three tumors, each with a maximum diameter of $45 \mathrm{~mm}$, and a sum tumor diameter $\leq 80 \mathrm{~mm}$, with a 5 -year survival rate of $75 \%{ }^{[7]}$. Also achieving a similar survival (71.2\%), came the up-to-seven criteria [the total of the size of the largest tumor (in $\mathrm{cm}$ ) and the number of tumors no larger than 7$]^{[8]}$, among others ${ }^{[9]}$.

According to cultural and religious particularities, deceased donor liver transplantation (DDLT) is precluded, or rarely performed in some Eastern countries. In that scenario, centers developed criteria to indicate LDLT for HCC patients. Tokyo has implemented the 5-5 rule (up to five nodules with a maximum diameter of $5 \mathrm{~cm}$ ), with an overall 5 -years survival rate of $75 \%^{[10]}$. The South Korea criteria expanded the Tokyo criteria to 6 nodules, with a 5-year survival rate of $76.3 \%{ }^{[3]}$. Choi et al. ${ }^{[11]}$ expanded the criteria for LDLT even more (up to seven tumors with the greatest diameter $\leq 70 \mathrm{~mm}$ ) reporting a 5 -year survival rate of $72 \%$.

Going further, it did not seem logical to restrict the indications only to size and number of nodules without considering the biological behavior of the tumor. Alpha-fetoprotein (AFP) level was included in a couple of studies, with different cutoff values - less than $400 \mathrm{ng} / \mathrm{mL}$, or less than $1000 \mathrm{ng} / \mathrm{mL}$ - and were associated with tumor number or diameter ${ }^{[12]}$. Shimamura et al. ${ }^{[10]}$ included AFP levels to select patients for LDLT, calling the 5-5-500 criteria (tumor size $\leq 5 \mathrm{~cm}$ diameter, $\leq 5$ nodules, AFP $\leq 500 \mathrm{ng} / \mathrm{mL}$ ), and achieved a 5 -year recurrence-free survival of $90 \%$.

Other markers are recently being included, such as des-gamma-carboxy prothrombin (DCP), also called protein induced by vitamin $\mathrm{K}$ absence or antagonist II. This marker was also associated with the presence of microvascular invasion ${ }^{[3]}$. DCP measurement was included in the Kyoto group LDLT criteria (the number of HCC nodules up to ten in addition to the largest diameter $\leq 5 \mathrm{~cm}$ and serum DCP level $\leq$ $400 \mathrm{mAU} / \mathrm{mL}$ ). The 5 -year disease-free and overall survival rates were 93 and $82 \%$, respectively ${ }^{[13]}$. In the same direction, Taketomi et al. ${ }^{[14]}$ included all HCCs with a diameter of $\leq 5 \mathrm{~cm}$ and DCP $<300 \mathrm{mAU} / \mathrm{mL}$, achieving a 5-year recurrence-free survival rate of $80 \%$. Both Hangzhou and Toronto groups included the HCC biopsy result in their selection criteria ${ }^{[12]}$.

The MoRAL score was developed in Korea to predict HCC recurrence after LDLT, and includes serum tumor markers, AFP and DCP. The MoRAL score has a high predictive power for tumor recurrence in 
patients transplanted with LDLT outside MC (c-statistic = 0.8). In fact, patients within MC and with high MoRAL scores had higher recurrence rates than patients outside MC and with a low MoRAL score ${ }^{[3]}$. When compared to other existent scores, it had the best performance in predicting tumor recurrence after LDLT. However, it is important to state that $70 \%-80 \%$ of HCC patients have hepatitis B virus infection in South Korea, so it is difficult to expand these results to Western countries, where hepatitis $\mathrm{C}$ dominates the etiologies for HCC and where most of the transplants are with deceased donors. It would be important to validate this model in the West, and for DDLT, to aid in the identification of highly selected patients beyond MC, who are at a low risk of tumor recurrence ${ }^{[15]}$.

To predict the risk of death after LT for HCC with DDLT, Mazzafero's group developed the Metroticket 2.0 score. They concluded that to achieve a 5-year survival of 70\%, AFP levels should be below $200 \mathrm{ng} / \mathrm{mL}$, and the sum of number and size (in $\mathrm{cm}$ ) of tumors should not exceed 7. These values change according to the AFP levels, and the prediction of survival after LT for HCC can be calculated online ${ }^{[8]}$.

The role of studying tumor biology (biopsy, tumor markers, tumor behavior) and the presence of microvascular invasion, which is a surrogate marker of worse survival ${ }^{[4]}$, could help in patient selection, selecting those patients with less aggressive tumors ${ }^{[16]}$. This approach could expand safely the indications for LT, keeping the expected 5-year survival above $70 \%{ }^{[3,8]}$.

In contrast to DDLT, recipient selection for LDLT is not limited by organ allocation systems. When the first results after LDLT for HCC were published, recurrence rates were higher than with DDLT. Several concerns were raised about the influence of liver regeneration and suboptimal oncological resection in LDLT, suggesting that it could affect recurrence ${ }^{[17]}$.

Recently, Zhang et al. ${ }^{[18]}$ conducted a meta-analysis comparing recurrence of HCC in patients transplanted with liver from living or deceased donors and found a higher recurrence rate of HCC in patients submitted to LDLT (HR 1.5). In the selected studies, 5-year recurrence-free survival in DDLT patients was between $42 \%$ and $100 \%$, and with living donors between $61.6 \%$ and $89 \%$. Waiting time was shorter for LDLT in the majority of the studies, which may be responsible for the higher recurrence rate in LDLT, speculating that patients with biologically higher risk tumors were selected. Selecting patients with a more aggressive tumor biology for LDLT, an effect of a shorter waiting list time and also expanding the criteria for LDLT are also factors that could have an influence on the reported higher recurrence rates in those patients.

Goldaracena et al. ${ }^{[19]}$ tried to answer the question regarding the observed higher recurrence rates in patients transplanted using living donors and conducted an intention to treat analysis. The patients were analyzed according to their inclusion status in the transplant list, as a potential LDLT (pLDLT) or potential DDLT (pDDLT). Patients were included if they fulfilled the Extended Toronto Criteria - total tumor volume $\leq 115 \mathrm{~m}^{3}$ and AFP $\leq 400 \mathrm{ng} / \mathrm{mL}$, no macrovascular invasion and no extrahepatic disease. The waiting list time was significantly shorter for patients listed as pLDLT, with better 5-year survival rates (68\% vs. 57\%). In the multivariate regression analysis, having a living donor available was a protective factor against death (HR 0.67). The survival benefit appeared to be predominantly in patients whose tumors fulfilled the MC: $70 \%$ in the pLDLT group and 53\% in the pDDLT group. Among patients beyond MC, 5-year survival rates were $62 \%$ for those outside MC in both groups. For those patients actually transplanted with a living donor, 5 -year recurrence-free survival rates were $80 \%$ vs. $72 \%$ for those actually transplanted with a deceased donor.

Results are still conflicting, but HCC recurrence and post-LT survival appear to correlate with biological tumor behavior and patient selection rather than if the patient was transplanted with a living or deceased donor. Reports from Eastern countries ${ }^{[3,9,13]}$, where the majority of patients are transplanted with living 
donors and with expanded criteria, show excellent disease-free and overall survivals. Even in the West, where there is a predominance of DDLT, the study by Goldaracena et al ${ }^{[19]}$ showed that having a living donor available had a protective effect, even if in the end, the patient is transplanted with a deceased donor.

As already stated, outcomes in LDLT for HCC can be affected by several factors, including time to transplant, the possibility to perform the transplant in expanded criteria patients, and the possibility of the liver regeneration adversely affecting tumor growth ${ }^{[20]}$.

Because of the limited number of deceased donors particularly in Asian countries, patients beyond MC were rarely submitted to DDLT. In the West, however, expanding LDLT can provide an alternative to DDLT, allowing many centers to indicate LT for patients beyond MC. Currently, organ allocation systems provide exception points for patients with early HCC, with favorable prognosis. As for recipients from living donors, organ availability is driven by the living donor. In that case, usually the transplant happens when the donor is ready, shortening the waiting time, allowing patients with more aggressive tumors to be transplanted. We are dealing with uneven criteria to indicate LT for HCC, defining these criteria not only on the basis of individual patient prognosis but by the fact of whether there is a living donor available.

If one looks at the results from more than a decade ago, it would be reasonable to address different selection criteria for patients undergoing DDLT or LDLDT. However, on the basis of recent research and a better understanding of tumor behavior, the discussion should be "how do we adequately select HCC patients for LT?", rather than to which transplant modality will the patient be submitted (LDLT, DDLT, Split Liver or Domino Transplantation).

Future directions are pointing to better patient selection - looking deeper into tumor biological behavior and addressing the presence of microvascular invasion, and not indicating LT to patients with aggressive tumors with a worse prognosis. It is urgent to validate existing models that predict survival in LDLT and DDLT, such as the MoRAL Score ${ }^{[3]}$, including such criteria in the organ allocation policy, currently restricted in many countries to the MC of $1996^{[6]}$. The practice reported by the Toronto group ${ }^{[19]}$, having the same criteria to indicate LT on the basis of patient prognosis, irrespectively if the patient will be assigned a living or deceased donor, follows the principle of equality. After being included in the waiting list, if there is a living donor available, the better it is for the patient and for the donor pool. If such a policy could be embraced by Western countries, perhaps LDLT in the West could reach comparable results as those achieved by our Asian fellows.

\section{DECLARATIONS}

\section{Authors' contributions}

The author contributed solely to the article.

\section{Availability of data and materials}

Not applicable.

\section{Financial support and sponsorship}

None.

\section{Conflicts of interest}

The author declared that there are no conflicts of interest.

\section{Ethical approval and consent to participate}

Not applicable. 


\section{Consent for publication}

Not applicable.

\section{Copyright}

(c) The Author(s) 2020.

\section{REFERENCES}

1. Kwong A, Kim WR, Lake JR, et al. OPTN/SRTR 2018 annual data report: liver. Am J Transplant 2020;20 Suppl s1:193-299.

2. Umeshita K, Eguchi S, Egawa H, et al. Liver transplantation in Japan: registry by the japanese liver transplantation society. Hepatol Res 2019;49:964-80.

3. Lee JH, Cho Y, Kim HY, et al. Serum tumor markers provide refined prognostication in selecting liver transplantation candidate for hepatocellular carcinoma patients beyond the Milan Criteria. Ann Surg 2016;263:842-50.

4. Bhatti ABH, Dar FS, Qureshi AI, et al. Living donor liver transplantation for hepatocellular carcinoma: a single-center experience from Pakistan. J Clin Exp Hepatol 2019;9:704-9.

5. Goldaracena N, Barbas AS. Living donor liver transplantation. Curr Opin Organ Transplant 2019;24:131-7.

6. Mazzaferro V, Regalia E, Doci R, et al. Liver transplantation for the treatment of small hepatocellular carcinomas in patients with cirrhosis. N Engl J Med 1996;334:693-9.

7. Yao FY, Xiao L, Bass NM, et al. Liver transplantation for hepatocellular carcinoma: validation of the UCSF-expanded criteria based on preoperative imaging. Am J Transplant 2007;7:2587-96.

8. Mazzaferro V, Llovet JM, Miceli R, et al. Predicting survival after liver transplantation in patients with hepatocellular carcinoma beyond the Milan criteria: a retrospective, exploratory analysis. Lancet Oncol 2009;10:35-43.

9. Toso C, Meeberg G, Hernandez-Alejandro R, et al. Total tumor volume and alpha-fetoprotein for selection of transplant candidates with hepatocellular carcinoma: a prospective validation. Hepatology 2015;62:158-65.

10. Shimamura T, Akamatsu N, Fujiyoshi M, et al; Japanese Liver Transplantation Society. Expanded living-donor liver transplantation criteria for patients with hepatocellular carcinoma based on the Japanese nationwide survey: the 5-5-500 rule - a retrospective study. Transpl Int 2019;32:356-68.

11. Choi HJ, Kim DG, Na GH, Hong TH, You YK. Extended criteria for living donor liver transplantation in patients with advanced hepatocellular carcinoma. Transplant Proc 2012;44:399-402.

12. Levi Sandri GB, Rayar M, Qi X, Lucatelli P. Liver transplant for patients outside Milan criteria. Transl Gastroenterol Hepatol $2018 ; 3: 81$.

13. Ito T, Takada Y, Ueda M, et al. Expansion of selection criteria for patients with hepatocellular carcinoma in living donor liver transplantation. Liver Transpl 2007;13:1637-44.

14. Taketomi A, Sanefuji K, Soejima Y, et al. Impact of des-gamma-carboxy prothrombin and tumor size on the recurrence of hepatocellular carcinoma after living donor liver transplantation. Transplantation 2009;87:531-7.

15. Chang Y, Cho Y, Lee JH, et al. Comparison of models for tumor recurrence after liver transplantation for the patients with hepatocellular carcinoma: a multicenter long-term follow-up study. Cancers 2019;11:1295.

16. Limkemann AJP, Abreu P, Sapisochin G. How far can we go with hepatocellular carcinoma in living donor liver transplantation? Curr Opin Organ Transplant 2019;24:644-50.

17. Fisher RA, Kulik LM, Freise CE, et al; A2ALL Study Group. Hepatocellular carcinoma recurrence and death following living and deceased donor liver transplantation. Am J Transplant 2007;7:1601-8.

18. Zhang HM, Shi YX, Sun LY, Zhu ZJ. Hepatocellular carcinoma recurrence in living and deceased donor liver transplantation: a systematic review and meta-analysis. Chin Med J 2019;132:1599-609.

19. Goldaracena N, Gorgen A, Doyle A, et al. Live donor liver transplantation for patients with hepatocellular carcinoma offers increased survival vs. deceased donation. $J$ Hepatol 2019;70:666-73.

20. Sugawara Y. Living-donor liver transplantation for patients with hepatocellular carcinoma in Japan: current situations and challenge. Hepatobiliary Pancreat Dis Int 2020;19:1-2. 